\title{
Does Metformin Treatment Influence Bone Formation in Patients with Nonalcoholic Fatty Liver Disease?
}

\author{
Authors \\ E. Soifer ${ }^{1}$, D. Gavish ${ }^{1,2}$, M. Shargorodsky ${ }^{2,3}$ \\ Affiliations \\ 1 Department of Medicine, Wolfson Medical Center, Tel Aviv, Israel \\ ${ }^{2}$ Sackler School of Medicine, Tel Aviv University, Tel Aviv, Israel \\ ${ }^{3}$ Department of Endocrinology, Wolfson Medical Center, Tel Aviv, Israel
}

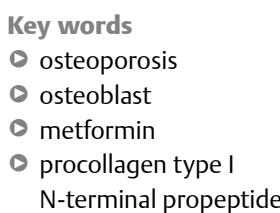

received 29.07.2014

accepted 12.11 .2014

Bibliography

DOI http://dx.doi.org/

10.1055/s-0034-1395652

Published online:

February 11, 2015

Horm Metab Res 2015;

47: 556-559

(c) Georg Thieme Verlag KG Stuttgart · New York

ISSN 0018-5043

\section{Correspondence}

\section{Shargorodsky, MD}

Wolfson Med Center

P.O. Box 5

Holon 58100

Israel

Tel.: + 972/3/5028378

Fax: + 972/3/5028375

marinas@wolfson.health.gov.il

\section{Abstract \\ $\nabla$}

Antidiabetic drug metformin that improves insulin sensitivity and used in the treatment of nonalcoholic fatty liver disease (NAFLD), may affect the bone health. Our study was designed to investigate a possible effect of metformin on bone formation marker, procollagen type I N-terminal propeptide (P1NP) in patients with NAFLD. In a randomized, placebo controlled study, 63 patients with NAFLD were assigned to one of 2 groups: Group 1 received daily metformin and Group 2 received placebo. Metabolic parameters, insulin resistance markers, and P1NP were determined. Although circulating P1NP levels

\section{Introduction}

$\nabla$

Accumulating evidence indicates that nonalcoholic fatty liver disease (NAFLD) is associated with low bone mass in both children and adult populations and may act as a risk factor for a osteoporosis-related bone fractures $[1,2]$. The pathogenesis of osteoporosis in patients with nonalcoholic fatty liver disease is multifactorial and still not completely understood. Recently it has been shown that circulating markers of bone metabolism, including osteoprotegerin, osteopontin, osteocalcin, and fetuin-A, have been altered in patients with NAFLD [3-5]. Moreover, antidiabetic drugs such as thiazolidinediones and metformin that improve insulin sensitivity and used in the treatment of NAFLD, may affect the bone health. Although most experimental data demonstrated that metformin has a direct osteogenic effect [6-8], the results of clinical trials are equivocal. Metformin treatment has been shown to reduce the association between diabetes and fractures in type 2 diabetic patients [9]. However, significant decrease in markers of bone formation in diabetic patients treated with metformin compared to rosiglitazone was found [10]. did not differ significantly between the groups at baseline, at the end of the study, P1NP was significantly lower in patients treated with metformin than in the placebo group $(\mathrm{p}<0.007)$. Within-group analysis indicated that P1NP levels significantly decreased $(p=0.023)$ in patients receiving metformin during 4-month follow-up period, while no change in P1NP was observed in placebo group $(p=0.359)$. In general linear model metformin treatment was the only significant independent predictor of endpoint P1NP. Metformin treatment was associated with decrease in P1NP levels in patients with NAFLD. The effect on P1NP was independent of glucose lowering effect and caused from exposure to metformin per se.

In recent years, bone turnover markers with better performance have been assessed. The use of $\mathrm{N}$-terminal propeptide of type 1 collagen, as a specific bone formation marker as well as reference analyte for bone turnover markers in clinical studies, was recommended by the International Osteoporosis Foundation and the International Federation of Clinical Chemistry and Laboratory Medicine (IFCC) [11]. Our study was designed to investigate a possible effect of metformin on bone formation marker, PINP, in a specific study population in which this issue was not studied yet, that is, in patients with nonalcoholic fatty liver disease.

\section{Subjects and Methods \\ $\checkmark$}

As previously reported, 63 patients (32 males and 31 females) diagnosed with nonalcoholic fatty liver disease were recruited from the outpatient clinic at the Wolfson Medical Center to participate in the study [12]. The diagnosis of NAFLD was based on the results of abdominal ultrasonography and exclusion of viral, autoimmune or drug induced liver disease as well as alcohol intake of more than $20 \mathrm{~g} /$ day. 
Patients with unbalanced endocrine disease or any disease that might affect absorption of medications were excluded, as were patients with plasma creatinine $>1.5 \mathrm{mg} / \mathrm{dl}$, and electrolyte abnormalities. Patients included in the study were stabilized on their previous medical treatment in the outpatient clinic for up to 3 months, with an effort not to change treatment during the study to prevent possible effects on the study parameters. None of the study participants had previously received metformin therapy. Patients were randomly assigned to one of the 2 groups: Group 1 received oral daily metformin at a dose of $850-1700 \mathrm{mg} /$ day and Group 2 received matching placebo capsules. Of the 63 patients recruited to the study, 52 completed the 4-month treatment period (27 from Group 1 and 25 from Group 2). The study was a randomized, placebo controlled. Metformin therapy was generally well tolerated; only one patient from metformin group withdrew because of gastrointestinal side effects. The reason for the rest of dropouts was loss to follow-up.

\section{Informed consent}

The study was approved by the regional ethical committee, and all procedures were performed in accordance with the guidelines of the Declaration of Helsinki. Informed written consent was obtained from all the subjects before participation. The study had been registered in ClinicalTrials.gov registry. The registration number: NCT01084486.

\section{Biochemical parameters}

Blood sampling for full chemistry and metabolic parameters, including fasting glucose, fasting insulin, lipid profile, CRP, fibrinogen, and liver function tests was performed at baseline and at the end of the study. The bone formation marker procollagen type I N-terminal propeptide (P1NP) were determined by electrochemiluminescence Immunoassaay (Roche). Adiponectin was determined by a commercial sandwich enzyme immunoassay technique [R\&D Systems, Minneapolis, USA (Catalogue number DRP300)] with $2.8 \%$ intra-assay and $6.5 \%$ inter-assay variability.

\section{Statistical analysis}

Analysis of data was carried out using SPSS 10.0 statistical analysis software (SPSS Inc., Chicago, IL, USA). For continuous variables, such as hemodynamic, biochemistry and arterial elasticity parameters, descriptive statistics were calculated and reported as mean \pm standard deviation. Normalcy of distribution of continuous variables was assessed using the Kolmogorov-Smirnov test (cutoff at $\mathrm{p}=0.01$ ). Categorical variables such as sex and co-morbidities were described using frequency distributions and are presented as frequency (\%). Additionally, univariate general linear modeling (GLM) was used to compare outcomes by treatment assignment controlling for values of covariates Within a given treatment group, the $t$-test for paired samples was used to compare before vs. post-treatment values of outcomes. Categorical variables were compared between groups using the chi-square test. All tests are 2 -sided and considered significant at $\mathrm{p}<0.05$.

\section{Results}

Clinical and demographic characteristics of the study groups are presented in $\odot$ Table 1 . As can be seen, the 2 groups were similar with respect to age, gender, presence of cardiovascular risk

Table 1 Demographic and clinical characteristics of study patients.

\begin{tabular}{|c|c|c|c|}
\hline Variables & Metformin-treated patients $(n=32)$ & Placebo group $(n=31)$ & p-Value \\
\hline Male/Female & $17 / 15$ & $14 / 17$ & 0.352 \\
\hline Age (years) & $51.9 \pm 10.9$ & $55.2 \pm 14.0$ & 0.306 \\
\hline $\operatorname{BMI}\left(\mathrm{kg} / \mathrm{m}^{2}\right)$ & $32.6 \pm 5.8$ & $31.5 \pm 5.6$ & 0.410 \\
\hline Current smokers, n (\%) & $4(12.5 \%)$ & $2(6.5 \%)$ & 0.391 \\
\hline Hypertension, $\mathrm{n}(\%)$ & $15(46.9 \%)$ & $15(48.4 \%)$ & 0.599 \\
\hline Dyslipidemia, n (\%) & $21(65.6 \%)$ & $14(45.2 \%)$ & 0.079 \\
\hline DM/IFG, n (\%) & $6 / 14(18.8 / 43.8 \%)$ & $2 / 13(6.5 / 41.9 \%)$ & 0.128 \\
\hline \multicolumn{4}{|l|}{ Concomitant medication } \\
\hline Antidiabetic treatment (\%) & $6(18.8 \%)$ & $2(6.5 \%)$ & 0.194 \\
\hline Statins (\%) & $18(56.3 \%)$ & $11(35.5 \%)$ & 0.079 \\
\hline ACEIs/ARBs (\%) & $9(28.1 \%)$ & $9(29.0 \%)$ & 0.609 \\
\hline Diuretics (\%) & $1(3.1 \%)$ & $6(19.4 \%)$ & 0.104 \\
\hline$\beta$-blockers (\%) & $9(28.1 \%)$ & $10(32.3 \%)$ & 0.784 \\
\hline CCB-blockers (\%) & $5(15.6 \%)$ & $8(25.8 \%)$ & 0.351 \\
\hline Aspirin (\%) & $13(40.6 \%)$ & $9(29.0 \%)$ & 0.292 \\
\hline Baseline systolic BP (mm Hg) & $138.8 \pm 17.6$ & $133.6 \pm 18.1$ & 0.258 \\
\hline Baseline diastolic BP $(\mathrm{mm} \mathrm{Hg})$ & $80.1 \pm 9.8$ & $74.6 \pm 13.5$ & 0.072 \\
\hline Baseline fasting glucose (mg/dl) & $131.8 \pm 51.3$ & $98.3 \pm 14.8$ & 0.034 \\
\hline Baseline total cholesterol (mg/dl) & $184.5 \pm 43.3$ & $191.1 \pm 37.0$ & 0.523 \\
\hline Baseline LDL Cholesterol (mg/dl) & $110.4 \pm 37.9$ & $112.7 \pm 34.2$ & 0.811 \\
\hline Baseline HDL-cholesterol (mg/dl) & $42.6 \pm 13.0$ & $48.2 \pm 15.2$ & 0.123 \\
\hline Baseline triglycerides (mg/dl) & $185.5 \pm 112.0$ & $143.1 \pm 63.2$ & 0.070 \\
\hline Baseline hs-CRP (mg/dl) & $0.9 \pm 1.1$ & $0.9 \pm 1.4$ & 0.969 \\
\hline Baseline AST (U/I) & $28.9 \pm 16.8$ & $28.0 \pm 8.2$ & 0.796 \\
\hline Baseline ALT (U/I) & $38.0 \pm 29.9$ & $32.6 \pm 15.3$ & 0.377 \\
\hline Baseline ALP (U/I) & $67.7 \pm 17.0$ & $72.2 \pm 23.5$ & 0.386 \\
\hline Baseline creatinine (mg/dl) & $0.9 \pm 0.1$ & $0.9 \pm 0.2$ & 0.723 \\
\hline Baseline HOMA-IR & $7.0 \pm 6.9$ & $5.4 \pm 4.9$ & 0.284 \\
\hline Baseline P1NP ( $\mu \mathrm{g} / \mathrm{l})$ & $39.8 \pm 16.4$ & $41.3 \pm 12.1$ & 0.754 \\
\hline
\end{tabular}


factors, and liver function. As can be seen from $\odot$ Table 1, both groups were similar at baseline in terms of P1NP levels, hemodynamic, and metabolic parameters. Although circulating P1NP levels were did not differ significantly between the groups at baseline $(p=0.754)$, at the end of the study, P1NP was significantly lower in patients treated with metformin than in the placebo group ( $\mathrm{p}<0.007)$ ( $\odot$ Fig. 1).

As shown in $\bullet$ Table 2, within-group analysis indicated that P1NP levels significantly decreased from $38.8 \pm 16.3$ to $26.0 \pm 9.6$ $(\mathrm{p}=0.023)$ in patients treated with metformin during 4-month follow-up period, while no change in P1NP was observed in placebo group ( $\mathrm{p}=0.359)$. Adiponectin levels tended to increase in metformin-treated patients, however, this increase did not reach statistical significance $(p<0.171)$. In placebo group adiponectin levels did not change during 4-month follow-up ( $\mathrm{p}<0.699$ ).

Systolic blood pressure (SBP) and diastolic blood pressure (DBP) did not change significantly during the follow-up in both study groups.

Significant declines in fasting glucose, triglycerides, and alkaline phosphatase together with a significant increase in HDL cholesterol were observed in metformin-treated patients. CRP and ALT decreased marginally during 4-month treatment period. Among metabolic parameters, ALT, AST, and alkaline phosphatase levels did not change in placebo group during 4-month follow-up.

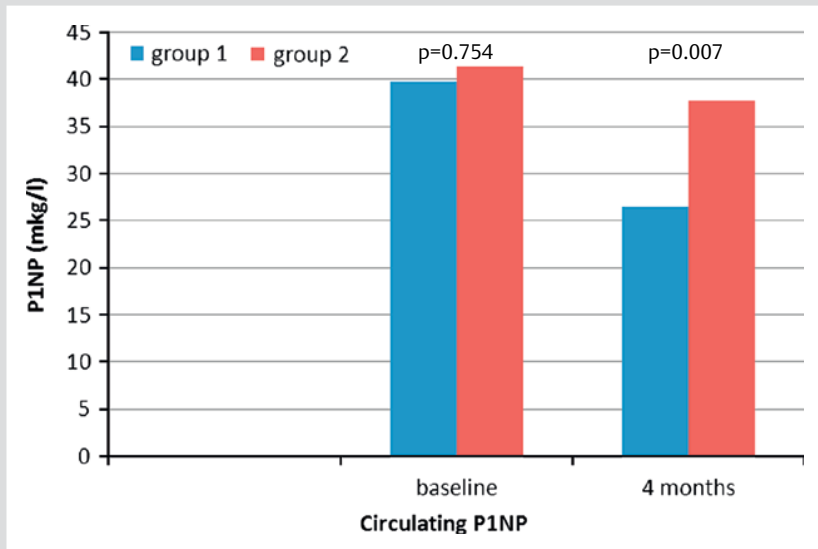

Fig. 1 Circulating P1NP by groups during 4-month follow-up. (Color figure available online only).

\section{Discussion}

$\nabla$

The present randomized, placebo-controlled study demonstrates that metformin treatment was associated with a significant decrease in P1NP levels in patients with nonalcoholic fatty liver disease. The effect on P1NP was independent of glucose lowering effect of metformin and caused from exposure to metformin per se.

To the best of our knowledge, the present study is the first to estimate a relation of insulin sensing effect of metformin to changes in bone remodeling as determined by P1NP level in patients with diagnosed NAFLD.

Findings of the present study concur with a published study, which showed significant reduction in serum markers of bone formation such as P1NP and bone alkaline phosphatase for both women and men with type 2 diabetes mellitus treated with rosiglitazone, metformin, and glyburide for 12 months, with the greatest changes in the metformin group (males: bone alkaline phosphatase, $-16.4 \%$, P1NP, $-19.3 \%$; females: bone alkaline phosphatase, $-15.7 \%$, P1NP, $-14.4 \%$ ) [13]. Moreover, it has been shown that in patients with poorly controlled type 2 diabetes, bone-specific alkaline phosphatase was decreased, whereas osteocalcin (OC) was increased after glycemic control for a month [14].

In the present study, the effect on P1NP was independent of glucose lowering effect of metformin. Since significant declines in fasting glucose were observed in metformin group, it has been questioned whether reduction in P1NP reflects the improvement in glucose homeostasis. To determine whether the endpoint measures of P1NP result from exposure to metformin per se, general linear model of endpoint P1NP was carried out. This analysis was modeled using multiple linear regression analysis with a backward, stepwise approach. For inclusion, the probability of $f$ was set at 0.05 for entry and at 0.10 for exclusion. Included in the model of endpoint P1NP were age, sex, and post-treatment fasting blood glucose. Additionally, metformin exposure was included as a fixed factor. In this model, group was the only significant independent predictor of endpoint P1NP ( $p=0.031)$. Recent experimental and clinical data have shown that metformin is osteogenic in vitro. It was reported that metformin can induce MC3T3-E1 osteoblastic cells differentiation and bone matrix synthesis via adenosine $5^{\prime}$-monophosphate-activated

Table 2 Change from baseline in homodynamic, arterial stiffness, and metabolic variables in each group.

\section{Variables}

Systolic BP $(\mathrm{mm} \mathrm{Hg})$

Diastolic BP $(\mathrm{mm} \mathrm{Hg})$

Creatinine $(\mathrm{mg} / \mathrm{dl})$

Urea (mg/dl)

Total Cholesterol (mg/dl)

Triglycerides (mg/dl)

HDL-cholesterol (mg/dl)

$\mathrm{LDL}$ Cholesterol (mg/dl)

$\operatorname{ALP}(\mathrm{U} / \mathrm{I})$

$\operatorname{ALT}(U / I)$

AST (U/I)

CRP $(\mathrm{mg} / \mathrm{dl})$

Fasting glucose (mg/dl)

HOMA-IR

Adiponectin $(\mathrm{ng} / \mathrm{ml})$

P1NP (mcg/l)

\begin{tabular}{ccc} 
& \multicolumn{3}{c}{ Metformin treated patients } \\
Baseline & 4-month & p-value \\
$139.0 \pm 17.9$ & $137.2 \pm 20.1$ & 0.565 \\
$80.6 \pm 9.2$ & $78.4 \pm 9.0$ & 0.403 \\
$0.9 \pm 0.1$ & $0.9 \pm 0.2$ & 0.481 \\
$32.3 \pm 10.5$ & $31.3 \pm 9.0$ & 0.569 \\
$179.3 \pm 40.3$ & $176.7 \pm 32.2$ & 0.722 \\
$195.4 \pm 119.1$ & $157.9 \pm 97.4$ & 0.033 \\
$41.3 \pm 12.2$ & $45.7 \pm 15.0$ & 0.001 \\
$103.5 \pm 37.3$ & $103.7 \pm 22.1$ & 0.979 \\
$66.5 \pm 17.8$ & $61.1 \pm 15.6$ & 0.007 \\
$38.5 \pm 31.7$ & $29.3 \pm 16.2$ & 0.092 \\
\hline $29.0 \pm 18.0$ & $25.4 \pm 9.7$ & 0.300 \\
$1.0 \pm 1.1$ & $0.5 \pm 0.4$ & 0.081 \\
$135.0 \pm 52.8$ & $115.8 \pm 27.0$ & 0.036 \\
$7.2 \pm 6.3$ & $7.3 \pm 10.9$ & 0.920 \\
$5662 \pm 2820$ & $6167 \pm 3265$ & 0.171 \\
$38.8 \pm 16.3$ & $26.0 \pm 9.6$ & 0.023
\end{tabular}

$\begin{array}{ccl}\text { Baseline } & \begin{array}{c}\text { Placebo group } \\ \text { 4-month }\end{array} & \text { p-value } \\ 133.0 \pm 19.5 & 139.8 \pm 19.3 & 0.091 \\ 73.3 \pm 14.1 & 75.0 \pm 9.4 & 0.589 \\ 0.9 \pm 0.2 & 0.9 \pm 0.2 & 0.627 \\ 31.2 \pm 7.6 & 31.1 \pm 10.5 & 0.960 \\ 190.0 \pm 38.5 & 186.2 \pm 32.6 & 0.616 \\ 140.8 \pm 58.0 & 135.0 \pm 57.6 & 0.588 \\ 47.8 \pm 14.3 & 49.6 \pm 15.3 & 0.329 \\ 110.8 \pm 35.2 & 109.0 \pm 25.7 & 0.786 \\ 74.3 \pm 24.6 & 67.4 \pm 19.6 & 0.065 \\ 34.9 \pm 15.8 & 29.7 \pm 16.3 & 0.120 \\ 29.4 \pm 8.4 & 27.4 \pm 8.3 & 0.246 \\ 1.0 \pm 1.5 & 0.5 \pm 0.5 & 0.139 \\ 98.1 \pm 15.9 & 103.9 \pm 18.5 & 0.015 \\ 5.3 \pm 5.1 & 6.1 \pm 8.0 & 0.525 \\ 10282 \pm 6610 & 10020 \pm 3265 & 0.699 \\ 41.3 \pm 12.1 & 37.8 \pm 13.9 & 0.359\end{array}$


protein kinase (AMPK) activation and subsequent induction of endothelial nitric oxide synthase (eNOS) and bone morphogenetic protein-2 (BMP-2) expression $[6,15]$. Metformin was also found to increase osteoblast proliferation, alkaline phosphatase activity and the number of mineralized nodules formed in rat primary osteoblasts, possibly via stimulation of Runx2 and IGF-1 production [16]. In contrast, 2 in vitro studies have shown no effect of metformin on the osteogenic differentiation of bone marrow-derived mesenchymal stem cells (MSCs) and matrix mineralization of both MC3T3-E1 cells and primary osteoblasts. Moreover, high concentration of metformin even clearly inhibited osteoblast differentiation $[17,18]$.

Less work has investigated the effect of metformin on bone in vivo, and the data are equivocal. It was reported that 2 months of treatment with metformin prevents the bone loss induced by ovariectomy in rats, suggesting protective effects of metformin against bone loss $[19,20]$. A very recent study performed in insulin-resistant mice also showed that metformin given for 6 weeks protects femoral bone architecture compared to rosiglitazone, although metformin had no effect on lumbar spine [21]. Nevertheless, it was shown that 1 - to 2 -month treatment with metformin, regardless of the administration route, has no significant effect on rodent bone architecture and fracture healing in vivo and that metformin significantly reduces bone formation rate in osteopenic trabecular bone [22]. Further, markers of bone formation were decreased in the metformin group compared to the rosiglitazone one in T2DM patients from the ADOPT study [13]. Given these discrepant findings, future studies should clarify the clinical impact of reduced P1NP during metformin treatment on bone density as well as fragility fractures in NAFLD patients.

Additionally, since the present study focused on P1NP assessment, the effect of metformin treatment on additional markers of osteoblastic differentiation such as osteocalcin as well as bone specific ALP will need to be evaluated by long-term studies in this population.

In conclusion, we have demonstrated that metformin treatment may affect bone formation in patients with nonalcoholic fatty liver disease. The findings of the present study justify further controlled, long-term studies with measurement of bone markers, bone density, and fractures rates to demonstrate overall clinical impact of decreased osteoblast-associated markers of bone formation in different populations, especially patients at increased risk for osteoporosis.

\section{Conflicts of Interest}

\section{$\nabla$}

The authors declare that they have no conflicts of interest in the authorship or publication of this contribution.

\section{References}

1 Li M, Xu Y, Xu M, Ma L, Wang T, Liu Y, Dai M, Chen Y, Lu J, Liu J, Bi Y, Ning G. Association between Nonalcoholic Fatty Liver Disease (NAFLD) and Osteoporotic Fracture in Middle-Aged and Elderly Chinese. J Clin Endocrinol Metab 2012; 97: 2033-2038

2 Pardee PE, Dunn W, Schwimmer JB. Non-alcoholic fatty liver disease is associated with low bone mineral density in obese children. Aliment Pharmacol Ther 2012; 35: 248-254

3 Syn WK, Choi SS, Liaskou E, Karaca GF, Agboola KM, Oo YH, Mi Z, Pereira TA, Zdanowicz M, Malladi P, Chen Y, Moylan C, Jung Y, Bhattacharya $S D$, Teaberry V, Omenetti A, Abdelmalek MF, Guy CD, Adams DH, Kuo $P C$, Michelotti GA, Whitington PF, Diehl AM. Osteopontin is induced by hedgehog pathway activation and promotes fibrosis progression in nonalcoholic steatohepatitis. Hepatology 2011; 53: 106-115
4 Haukeland JW, Dahl TB, Yndestad A, Gladhaug IP, Løberg EM, Haaland T, Konopski Z, Wium C, Aasheim ET, Johansen OE, Aukrust P, Halvorsen $B$, Birkeland KI. Fetuin A in nonalcoholic fatty liver disease: in vivo and in vitro studies. Eur J Endocrinol 2012; 166: 503-510

5 Fernández-Real JM, Ortega F, Gómez-Ambrosi J, Salvador J, Frühbeck G, Ricart $W$. Circulating osteocalcin concentrations are associated with parameters of liver fat infiltration and increase in parallel to decreased liver enzymes after weight loss. Osteoporos Int 2010; 21: 2101-2107

6 Kanazawa I, Yamaguchi T, Yano S, Yamauchi M, Sugimoto T. Metformin enhances the differentiation and mineralization of osteoblastic MC3T3-E1 cells via AMP kinase activation as well as eNOS and BMP-2 expression. Biochem Biophys Res Commun 2008; 375: 414-419

7 Jang WG, Kim EJ, Bae IH, Lee KN, Kim YD, Kim DK, Kim SH, Lee CH, Franceschi RT, Choi HS, Koh JT. Metformin induces osteoblast differentiation via orphan nuclear receptor SHP-mediated transactivation of Runx2. Bone 2011; 48: 885-893

8 Zhen $D$, Chen $Y$, Tang $X$. Metformin reverses the deleterious effects of high glucose on osteoblast function. J Diabetes Complications 2010; 24: 334-344

9 Vestergaard P, Rejnmark L, Mosekilde L. Relative fracture risk in patients with diabetes mellitus, and the impact of insulin and oral antidiabetic medication on relative fracture risk. Diabetologia 2005; 48: 1292-1299

10 Zinmam B, Haffner SM, Herman WH, Holman RR, Lachin JM, Kravitz BG Paul G, Jones NP, Aftring RP, Viberti G, Kahn SE.and the A Diabetes Outcome Progression Trial Study Group. Effect of rosiglitazone, metformin and glyburide on bone biomarkers in patients with type 2 diabetes. J Clin Endocrinol Metab 2010; 95: 134-142

11 Vasikaran S, Eastell R, Bruyère O, Foldes AJ, Garnero P, Griesmacher A, McClung M, Morris HA, Silverman S, Trenti T, Wahl DA, Cooper C, Kanis JA.IOF-IFCC Bone Marker Standards Working Group. Markers of bone turnover for the prediction of fracture risk and monitoring of osteoporosis treatment: a need for international reference standards. Osteoporos Int 2011; 22: 391-420

12 Sofer E, Boaz M, Matas Z, Mashavi M, Shargorodsky M. Treatment with insulin sensitizer metformin improves arterial properties, metabolic parameters, and liver function in patients with nonalcoholic fatty liver disease: a randomized, placebo-controlled trial. Metabolism 2011; 60: 1278-1284

13 Zinmam B, Haffner SM, Herman WH, Holman RR, Lachin JM, Kravitz BG Paul G, Jones NP, Aftring RP, Viberti G, Kahn SE. and the A Diabetes Outcome Progression Trial Study Group. Effect of rosiglitazone, metformin and glyburide on bone biomarkers in patients with type 2 diabetes. J Clin Endocrinol Metab 2010; 95: 134-142

14 Kanazawa I, Yamaguchi T, Yamamoto M, Yamauchi M, Kurioka S, Yano S, Sugimoto T. Serum osteocalcin level is associated with glucose metabolism and atherosclerosis parameters in type 2 diabetes mellitus. J Clin Endocrinol Metab 2009; 94: 45-49

15 Cortizo AM, Sedlinsky C, McCarthy AD, Blanco A, Schurman L. Osteogenic actions of the anti-diabetic drug metformin on osteoblasts in culture. Eur J Pharmacol 2006; 536: 38-46

16 Shah M, Kola B, Bataveljic A, Arnett TR, Viollet B, Saxon L, Korbonits M, Chenu $C$. AMP-activated protein kinase (AMPK) activation regulates in vitro bone formation and bone mass. Bone 2010; 47: 309-319

17 Wu W, Ye Z, Zhou Y, Tan WS. AICAR, a small chemical molecule, primes osteogenic differentiation of adult mesenchymal stem cells. Int J Artif Organs 2011; 34: 1128-1136

18 Kasai T, Bandow K, Suzuki H, Chiba N, Kakimoto K, Ohnishi T, Kawamoto $S$, Nagaoka E, Matsuguchi T. Osteoblast differentiation is functionally associated with decreased AMP kinase activity. J Cell Physiol 2009; 221: 740-749

19 Gao Y, Li Y, Xue J, Jia Y, Hu J. Effect of the anti-diabetic drug metformin on bone mass in ovariectomized rats. Eur J Pharmacol 2010; 635: 231-236

20 Mai QG, Zhang ZM, Xu S, Lu M, Zhou RP, Zhao L, Jia CH, Wen ZH, Jin $D D$, Bai XC. Metformin stimulates osteoprotegerin and reduces RANKL expression in osteoblasts and ovariectomized rats. J Cell Biochem 2011; 112: 2902-2909

21 Wang C, Li H, Chen SG, He JW, Sheng CJ, Cheng XY, Qu S, Wang KS, Lu $M L, Y u$ YC. The skeletal effects of thiazolidinedione and metformin on insulin-resistant mice. J Bone Miner Metab 2012; 30: 630-637

22 Jeyabalan J, Viollet B, Smitham P, Ellis SA, Zaman G, Bardin C, Goodship A, Roux JP, Pierre M, Chenu C. The anti-diabetic drug metformin does not affect bone mass in vivo or fracture healing. Osteoporos Int 2013; 24: 2659-2670 